\title{
FORCE AND POSITION CONTROL FOR ELECTROHYDRAULIC SYSTEMS OF A ROBOTIC EXCAVATOR
}

\author{
Q. P. Ha, Q. H. Nguyen, D. C. Rye, and H. F. Durrant-Whyte \\ Australian Centre for Field Robotics \\ Department of Mechanical and Mechatronic Engineering .J07 \\ The University of Sydney 2006 NSW, Australia \\ \{quang.ha@mech.eng.usyd.edu.au\}
}

\begin{abstract}
In impedance control of hydraulic excavators the piston position and ram force of each hydraulic cylinder for the axis control of the boom, arm, and bucket can be determined. The problem is thus how to find the control voltage applied to the servovalves to track these commands to the hydraulic systems. This paper presents analytic, simulation and experimental results for controllers that have been developed in our laboratory to achieve force and position tracking of electrohydraulic systems of a robotic mini-excavator. The systems with hydraulic cylinders as actuators are represented by a comprehensive model taking into account friction, nonlinearities, and uncertainties. A discontinuous observer is developed for estimating both piston velocity and disturbance force including friction. With an observer-based compensation for disturbance, tracking of the piston ram force and position is guaranteed using a robust sliding mode controller. The control signal consists of three components: equivalent control, switching control, and fuzzy control. High performance and strong robustness can be obtained as demonstrated by simulation and experiments performed on a hydraulically-actuated Komatsu PC05-7 robotic excavator. Promising results are reported, and issues relating to future work are discussed.
\end{abstract}

Keywords: force and position control, electrohydraulic systems, discontinuous observer, robust sliding mode control, robotic excavator.

\section{INTRODUCTION}

The Australian Centre for Field Robotics (ACFR) is conducting research into the area of autonomous earth-moving vehicles [1]. One objective of the research is to design control systems that allow for the automation of hydraulic excavators and other similar earthmoving machines. The excavator used for experiments in our laboratory is a Komatsu PC057 mini-excavator. The excavator is effectively a hydraulically-actuated robot with eight powered axes: two rubber tracks, cab slew, boom swing, boom, arm, bucket, and a small blade for back filling. The machine is extensively instrumented with joint angle encoders, pressure transducers and two-axis load pins. Figure 1 shows the current robotic excavator with its supplementary instrumentation.

Our research towards autonomous excavation concentrates on the execution of common tasks such as digging building footings, or loading haul trucks from an open-cut mine bench, and to understand the nature of tool soil interactions. As the bucket comes in contact with its environment, the contact force must be regulated such that it remains within a specific range. Impedance control has been proposed for robotic excavators [2] to achieve a desired dynamic relationship between the digging force and the bucket tip position given an excavation task in the world space. In impedance control of hydraulic excavators the ram forces of each hydraulic cylinder for the axis control of the boom, arm, and bucket can be determined. The problem is thus how to find the control voltage applied to the servovalves to track these desired ram forces. Various control methods have been developed in the robotics community to meet this requirement. This paper presents a new technique, based on variable structure systems, that has been developed in our laboratory to achieve robustness in force and position tracking of electrohydraulic systems of our mini-excavator while implementing impedance control for the excavator dynamics. The systems with linear actuators are represented by a comprehensive model taking into account friction, nonlinearities, and uncertainties. A discontinuous observer is developed for estimating both piston displacement velocity and disturbance 
including load force and friction. With an observerbased compensation for the disturbance force, tracking the piston ram force and position is guaranteed using a robust sliding mode controller. The control signal consists of three components: equivalent control, switching control, and fuzzy control. High performance and strong robustness can be obtained as demonstrated by simulation and experiments performed on a hydraulically-actuated Komatsu PC05-7 mini-excavator.

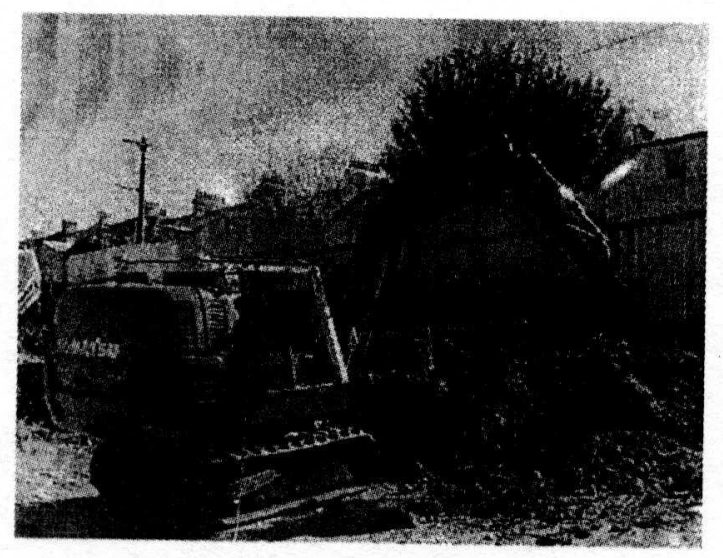

Figure 1. Experimental robotic excavator.

\section{ELECTROHYDRAULIC SERVO MODELLING.}

The position control of hydraulic systems has been studied in [3], where classical PID controllers are proposed. This traditional approach is based on local linearisation of the nonlinear dynamics about a nominal operating point. The effectiveness of such a controller may easily be understood from a linearised model of the hydraulic drive system. For the axis control of our robotic excavator, this linearised model proved to be very useful in gaining physical insight into the behaviour of the whole system. For simplicity, fixed-gain PID controllers were initially proposed for joint position control. The controllers were subsequently implemented for each of the excavator's axes, and were experimentally evaluated. Promising results were obtained, showing very accurate position control of the excavator arm in free motion. Our preliminary experiments, however, also identified a need for more robust control strategies, particularly during the force-constrained motion of the excavator bucket when interacting with the soil. Implementing impedance control to excavator dynamics [2] requires the ram force generated at each cylinder of the excavator arms follow a desired function of time when executing digging tasks. Nonlinear effects occurring during this interaction, and in the hydraulic system itself, complicate the control strategy requirements. It is known gravitational and friction between the piston and cylinder should be compensated for to achieve high performance of heavy-duty hydraulic machines, such as excavators [4]. Furthermore, oil viscosity, oil flow through the hydraulic servo-valve, and variable loading, will cause hydraulic control systems to suffer from highly nonlinear time-variant dynamics, load sensitivity, and parameter uncertainty. Thus, these factors have to be taken into account in servo hydraulic modelling and control.

The hydraulic actuators incorporated in the blade, boom swing, boom, arm, and bucket attachments of the excavator are axial hydraulic cylinders. The flow of hydraulic oil to the cylinder is regulated by a direct drive servo valve with an electrically-controlled closed loop that controls spool position. This system could be generally described by a six-order differential equation. For simplicity, the following linear expression can be used with little loss of accuracy for frequencies up to $200 \mathrm{~Hz}$ :

$$
x_{v}=K_{v} u
$$

where $x_{v}$ is the spool valve displacement and $u$ is the valve input voltage. Thus, a nonlinear state model can be obtained as in [5], based on the relationship between the valve displacement $x_{v}$ and the fluid flow to the head side $Q_{1}$ and from the rod side $Q_{2}$, the continuity of flow in the cylinder, and the force balance equation for the piston:

$$
\begin{aligned}
& \dot{x}=v \\
& \dot{v}=\frac{1}{M}\left[A_{1} P_{1}-A_{2} P_{2}-F_{L}\right] \\
& \dot{P_{1}}=\frac{\beta_{e}}{A_{1} x+V_{h}}\left\{K u\left[s\left(x_{v}\right) \sqrt{P_{S}-P_{1}}+s\left(-x_{v}\right) \sqrt{P_{1}}\right]\right. \\
& \left.\quad-C_{i p}\left(P_{1}-P_{2}\right)-A_{1} v\right\}
\end{aligned}
$$

where $x$ is the piston displacement $[m], v$ is its velocity, $P_{1}$ and $P_{2}$ are respectively the fluid pressures at the head and rod sides of the cylinder $[P a], u$ is the control input $[V], F_{L}$ is a load disturbance on cylinder including external forces and friction $[N], P_{S}$ is the supply pressure $[P a], K$ is a fixed constant, $M$ is the equivalent moving mass $[\mathrm{kg}], C_{i p}$ is the internal leakage coefficient, and $s\left(x_{v}\right)$ is a switching function depending on the extension or retraction of the piston [5]:

$$
s\left(x_{v}\right)=\left\{\begin{array}{ll}
1 & x_{v} \geq 0 \\
0 & x_{v}<0
\end{array} .\right.
$$


By assigning the ram force

$$
F=A_{1} P_{1}-A_{2} P_{2}
$$

as a state variable, an equivalent form of (2) can be obtained as follows

$$
\begin{aligned}
& \dot{x}=v \\
& \dot{v}=\frac{1}{M}\left[F-F_{L}\right] \\
& \dot{F}=a_{12} v+a_{13} F+a_{14} P_{1}+b_{1} u \\
& \dot{P}_{1}=a_{22} v+a_{23} F+a_{24} P_{1}+b_{2} u,
\end{aligned}
$$

where

$$
\begin{aligned}
a_{12} & =-\left(\frac{A_{1}^{2} \beta_{e}}{A_{1} x+V_{h}}+\frac{A_{2}^{2} \beta_{e}}{A_{2}(L-x)+V_{h}}\right), \\
a_{13} & =-\left(\frac{A_{1} / A_{2}}{A_{1} x+V_{h}}+\frac{1}{A_{2}(L-x)+V_{h}}\right) \beta_{e} C_{i p}, \\
a_{14} & =a_{13}\left(A_{2}-A_{1}\right), \\
a_{22} & =-\frac{A_{1}^{2} \beta_{e}}{A_{1} x+V_{h}}, a_{23}=-\frac{1 / A_{2}}{A_{1} x+V_{h}} \beta_{e} C_{i p}, \\
a_{24} & =a_{23}\left(A_{2}-A_{1}\right), \\
b_{1} & =\frac{A_{1} \beta_{e} K}{A_{1} x+V_{h}}\left[s\left(x_{v}\right) \sqrt{P_{S}-P_{1}}+s\left(-x_{v}\right) \sqrt{P_{1}}\right]+ \\
& +\frac{A_{2} \beta_{e} K}{A_{2}(L-x)+V_{h}}\left[s\left(x_{v}\right) \sqrt{P_{2}}+s\left(-x_{v}\right) \sqrt{P_{S}-P_{2}}\right], \\
b_{2} & =\frac{A_{1} \beta_{e} K}{A_{1} x+V_{h}}\left[s\left(x_{v}\right) \sqrt{P_{S}-P_{1}}+s\left(-x_{v}\right) \sqrt{P_{1}}\right] .
\end{aligned}
$$

In (3) information of the states can be obtained from the piston position and pressure transducers. Our experiments indicated however that differential pressure readings would be inadequate when used to represent the external force $F$ exerted on the hydraulic piston. Taking static friction into account, load pins inserted at the cylinder rod eyes are used for force measurements in our mini-excavator for a more accurate representation of the force $F$ [4]. The piston velocity, $v$, and load disturbance $F$ will be estimated using a discontinuous observer as described in the next section.

Remark 1: For simplicity the cylinder can be considered effectively a double-rod cylinder. In that case, the piston effective area is $A=A_{1}=A_{2}$, and if $P_{1}+P_{2}=P_{S}$, the supply pressure, the hydraulic servo model (3) can be reduced to a third-order system as described in [6].

Numerical values of parameters for the excavator arm-axis hydraulic control system can be found in [7].

\section{DISCONTINUOUS OBSEVER FOR DISTURBANCE AND VELOCITY ESTIMATION}

In tracking control of electrohydraulic manipulators there is a need to compensate for the influence of external disturbance such as the acting force coming from outside the hydraulic cylinder, gravity, and friction. Hydraulic servos are affected by a variety of frictional forces including linear and nonlinear viscous frictions and Coulomb friction. An adaptive nonlinear observer [8] is proposed to compensate for Coulomb friction in tracking control of a manipulator actuated by a linear hydraulic cylinder. Simultaneous estimation of velocity and Coulomb friction [5] is obtained via the observer where velocity is estimated independently using a first order low-pass filtered differentiator and static friction is taken into consideration using an approximation model. Variable structure control theory has brought a new insight to nonlinear observers in term of high insensitivity to uncertainties [9]. It is from this merit that a discontinuous observer is developed to provide robust estimate of the piston velocity and disturbance force. Assuming that the disturbance force is slowly time-varying, the state model for can be written as

$$
\dot{v}=w-F_{L}, \dot{F}_{L}=0, \dot{x}=v,
$$

with the output $y=x$, where $w$ is the acting force measured by a force sensor.

Remark 2: In [8], $w$ denotes the force due to all sources other than friction. The ram force (4) is used to represent this force for estimating friction in a servo hydraulic system [5] where the influence of gravity and external disturbance can be negligible. For hydraulic systems of the excavator, force measurements from load pins are employed in our application to provide estimate of the disturbance force $F$.

The Utkin observer [10] for (6) is described by

$$
\begin{aligned}
& \left(\begin{array}{l}
\dot{\hat{v}} \\
\dot{\hat{F}}_{L}
\end{array}\right)=\mathbf{A}_{11}\left(\begin{array}{l}
\hat{v} \\
\hat{F}_{L}
\end{array}\right)+\mathbf{B}_{1} w+\mathbf{L} \sigma \\
& \dot{\hat{y}}=\mathbf{A}_{21}\left(\begin{array}{l}
\hat{v} \\
\hat{F}_{L}
\end{array}\right)-\mathbf{L} \sigma,
\end{aligned}
$$

where $\sigma=\mu \operatorname{sgn}\left(e_{y}\right)$ with $\mu>0$ and $e_{y}=\hat{y}-y$ is the output error, the circumflex $\left({ }^{\wedge}\right)$ denotes the estimates, $\quad \mathbf{A}_{11}=\left(\begin{array}{cc}0-1 \\ 0 & 0\end{array}\right), \quad \mathbf{A}_{21}=\left(\begin{array}{ll}1 & 0\end{array}\right), \quad \mathbf{B}_{1}=\left(\begin{array}{l}1 \\ 0\end{array}\right)$, 
and $\mathbf{L}=\left(\begin{array}{l}l_{1} \\ l_{2}\end{array}\right)$ is the observer gain to be determined. Let the observer error be $\mathbf{e}_{x}=\left(\begin{array}{l}\hat{v} \\ \hat{F}_{L}\end{array}\right)-\left(\begin{array}{l}v \\ F_{L}\end{array}\right)$, and $\mathbf{e}=\mathbf{e}_{x}+\mathbf{L} e_{y}$, by taking the time derivatives one can obtain

$$
\begin{gathered}
\dot{\mathbf{e}}=\left(\mathbf{A}_{11}+\mathbf{L} \mathbf{A}_{21}\right) \mathbf{e}-\left(\mathbf{A}_{11}+\mathbf{L} \mathbf{A}_{21}\right) \mathbf{L} e_{y}, \\
\dot{e}_{y}=\mathbf{A}_{21} \mathbf{e}-\mathbf{A}_{21} \mathbf{L} e_{y}-\mu \operatorname{sgn}\left(e_{y}\right) .
\end{gathered}
$$

Since the pair $\left(A_{11}, A_{21}\right)$ is observable, $L$ can be chosen such that the matrix $\left(\Lambda_{11}+\mathbf{L A}_{21}\right)$ has a desired stable eigenstructure. It can be shown from (9) that for large enough $\mu$, a sliding motion can be induced on the output error after some finite time $t_{s}$. It follows that for all subsequent time $t>t_{s}, e_{y}=0$ and $\dot{e}_{y}=0$. Thus (8) becomes

$$
\dot{\mathbf{e}}=\left(\mathbf{A}_{11}+\mathbf{L} \mathbf{A}_{21}\right) \mathbf{e},
$$

and $\mathbf{e} \rightarrow 0$ as $t \rightarrow \infty$ with a convergence rate depending on a proper choice of the observer gain $\mathbf{L}$ or, in other words, on selecting eigenvalues of the following observer characteristic equation:

$$
\operatorname{det}\left(s \mathbf{I}-\mathbf{A}_{11}-\mathbf{L} \mathbf{A}_{21}\right)=s^{2}-l_{1} s+l_{2}=0 .
$$

The schematic diagram of the proposed observer is shown in Figure 2. In order to reduce chattering associated with the sliding motion of the output error, the signum function $\sigma=\mu \operatorname{sgn}\left(e_{y}\right)$ in (9) is replaced by a sigmoidal one resulted from a fuzzy reasoning technique presented in [11]:

$$
\sigma=\mu \tanh \left(e_{y} / \gamma_{e}\right)
$$

where $\gamma_{e}$ is some positive constant.

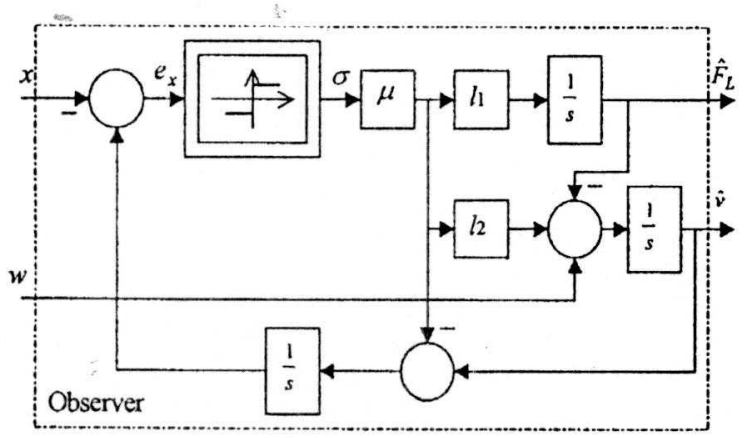

Figure 2. Observer schematic diagram

\section{CONTROLLER DESIGN}

Various advanced control methods addressing the electrohydraulic servo control problem have been reported in literature. Among these methods, variable structure control with a sliding mode has been promoted by many authors [6,12-14]. The reason is mainly because of its robustness to uncertaintics. Different sliding mode controllers have been derived in our laboratory, based on position control and on both position and differential pressure control. In accordance with the proposed impedance control scheme for excavator dynamics [2], force and position tracking are focused in this paper.

Given the nonlinear dynamic equations in (2), the control objective is to design a chattering-attenuated sliding mode controller that provides robust tracking of the desired ram force $F_{r}$ and piston position $x_{r}$ required to implement impedance control of the excavator bucket tip in the presence of soil uncertainties. Let us define the control errors

$$
e_{1}=x-x_{r}, e_{2}=v-v_{r}, e_{3}=F-F_{r}, e_{4}=P_{1}-P_{1 r} \text {, }
$$

where $v_{r}$ and $P_{1 r}$ are respectively the desired piston velocity and head-side fluid pressure.

Assumption l: Estimates of the piston velocity and disturbance force obtained from the observer (7) are adequately accurate.

From (5) and (12) the following nonlinear dynamics can be derived for the error vector $\mathbf{c}=\left[\begin{array}{llll}e_{1} & e_{2} & e_{3} & e_{4}\end{array}\right]^{T}$ :

$$
\dot{\mathbf{e}}=\mathbf{A}\left(\mathbf{x}, \mathbf{x}_{r}\right)+\mathbf{B}(u+f),
$$

where

$$
\mathbf{A}\left(\mathbf{x}, \mathbf{x}_{r}\right)=\left(\begin{array}{l}
\hat{v}-v_{r}=e_{2} \\
\frac{F-\hat{F}_{L}}{M}-\dot{v}_{r}=\frac{e_{3}-\hat{F}_{l}}{M} \\
a_{12} \hat{v}+a_{13} F+a_{14} P_{1}-\dot{F}_{r} \\
a_{22} \hat{v}+a_{23} F+a_{24} P_{1}-\dot{P}_{1 r}
\end{array}\right), \mathbf{B}=\left(\begin{array}{l}
0 \\
0 \\
b_{1} \\
b_{2}
\end{array}\right),
$$

and $f$ is an uncertain source taking into account parameter variations and modelling mismatch.

Assumption 2: $f$ is unknown but bounded by a known positive function $\rho_{s w}$ :

$$
|f| \leq \rho_{s w} .
$$


Let us now define the following switching function

$$
S=\mathrm{Ce}=e_{3}+c_{2} e_{2}+c_{1} e_{1},
$$

where $\mathbf{C}=\left[\begin{array}{llll}c_{1} & c_{2} & 1 & 0\end{array}\right]$ and $c_{i}(i=1,2)$ are positive constants to be specified according to the desired dynamics of the closed-loop system. From (15) it can be derived that the sliding mode $S=0$ is associated with the eigenvalues of the following characteristic equation:

$$
\frac{1}{M} s^{2}+c_{2} s+c_{1}=0 \text {. }
$$

Remark 3: With the choice of the sliding function (17) the influence of the pressure error $e$ can be excluded.

A necessary condition for the state trajectory to stay on the sliding mode $S=0$ is $\dot{S}=0$. The following control law [15]

$$
u=u_{e q}+u_{s w}+u_{f t}
$$

is proposed. It consists of three components: an equivalent control $u_{e q}$ to assign desired dynamics to the closed-loop system, a switching control $u_{s w}$ to guarantee a sliding mode, and a fuzzy control $u_{f}$ to enhance fast tracking and to attenuate chattering. The equivalent control can be obtained from the nominal system parameters, denoted with bars $\left({ }^{-}\right)$, as follows $[6,15]$ :

$$
\begin{aligned}
& u_{e q}=-(\mathrm{CB})^{-1}(\mathrm{CA}) \\
& =-\bar{b}_{1}^{-1}\left[\left(c_{1}+\bar{a}_{12}\right) \hat{v}+\left(\frac{c_{2}}{M}+\bar{a}_{13}\right) F+\bar{a}_{14} P_{1}-r-\frac{c_{2}}{M} \hat{F}_{L}\right],
\end{aligned}
$$

where $r=c_{1} \dot{x}_{r}+c_{2} \ddot{x}_{r}+\dot{F}_{r}$ and the coefficients $c$ $(i=1,2)$ are chosen according to the desired characteristic roots of (18).

Remark 4: The law (19) can be considered as a combination of state feedback and feedforward control. Experience shows that the observer dynamics (11) should be chosen 3-5 times as faster as the system dynamics (18).

The switching control and fuzzy control are respectively given by [15]:

$$
\begin{gathered}
u_{s w}=-\rho_{s w} \operatorname{sgn}(\varphi), \\
u_{f t}=-u_{f m} \tanh \left(\varphi / \gamma_{t}\right),
\end{gathered}
$$

where $\varphi=S C B=S b_{1}$, and $u_{m}$ and $\gamma_{1}$ are some positive constants.

Remark 5: The law (21) is to force the system trajectory back to the sliding mode in the presence of uncertainties while that of $(22)$ is to smooth out the control action.

\section{SIMULATION RESULTS}

Consider multi-axis control of the experimental excavator arm actuated by the hydraulic servo system described in [6]. The desired eigenvalues are chosen at $\{-10,-10\}$, which gives a settling time of $0.4 \mathrm{~s}$ and no overshoot. The controller parameters are: $l_{1}=-70$, $l_{2}=2500, \mu=5, \gamma_{e}=0.01, c_{1}=100, c_{2}=20, \rho_{s w}=3$, $u_{m}=3$, and $\gamma=0.1$. The fourth-order Runge-Kutta method with a time step of $1 \mathrm{~ms}$ is used in our simulation. We consider first the free motion (no load) tracking of a step input; for example, stroking the arm cylinder from $0.144 \mathrm{~m}$ to $0.070 \mathrm{~m}$. Good tracking performance and load insensitivity can be observed in Figure 3 where the step response with the proposed control technique is presented.

In impedance control of the robotic excavator, our objectives focus on the execution of common excavation tasks such as digging building footings, or loading haul trucks from an open-cut mine bench. We are currently concentrating on low-level control, and particularly on understanding the nature of interaction between the excavator and the ground. Let us consider next the practical problem of digging a certain soil, for example, "sandy loam". For our experimental mini-excavator the digging force for a cut depth of about $0.2 \mathrm{~m}$ can be estimated [16] to be about $3.4 \mathrm{kN}$. When rotary cutting with the excavator arm, the arm cylinder piston has to track square motion sequences between $0.07 \mathrm{~m}$ and $0.17 \mathrm{~m}$. The tracking responses with the proposed controller are shown in Figure 4.

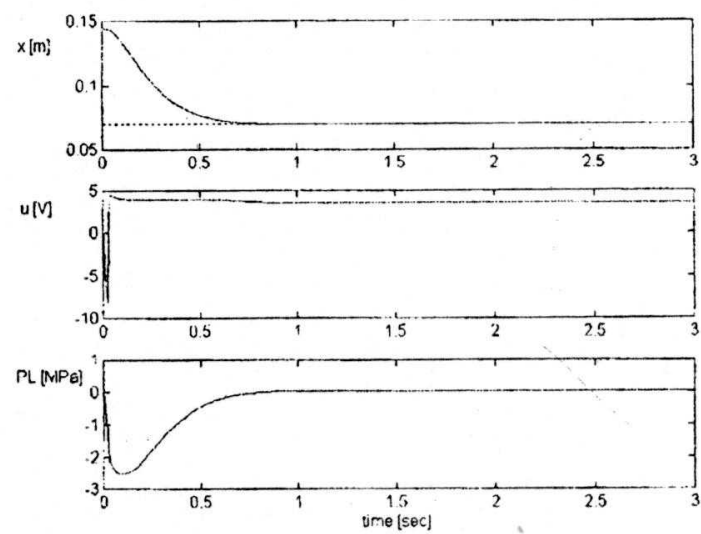

Figure 3. Free space tracking response 

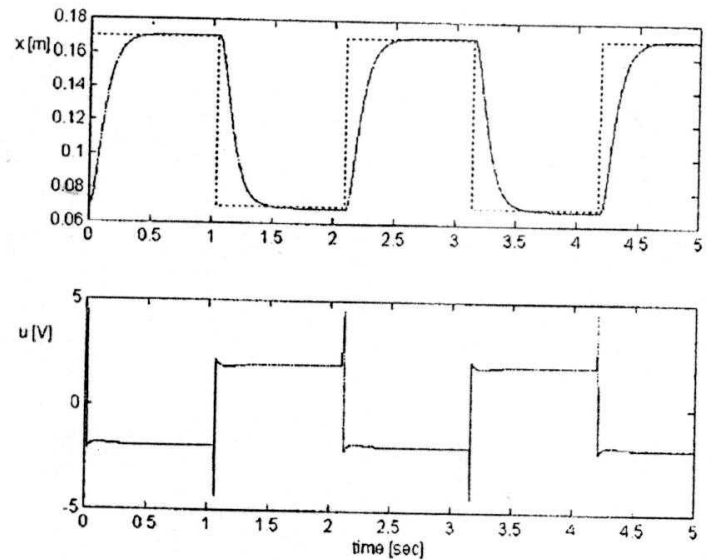

Figure 4. Rotary cutting tracking responses

It can be seen that robust tracking and a significant reduction of chattering are obtained with the proposed control scheme. The superiority of the technique has also been demonstrated through extensive simulations for a typical servo electrohydraulic system in the cases of severe uncertainties, such as large variations of parameters and load, and nonlinear friction, as reported in [6].

\section{EXPERIMENTAL RESULTS}

Experimental work has been performed on a Komatsu PC05-7 mini-excavator (Figure 1). The machine's original manual direction control valves have been removed and replaced by Moog electrohydraulic servo valves. Additional ancillary equipment needed to support the servo valves are an accumulator with unloading valve, solenoid check valves and an oil to air radiator. The hydraulic system is instrumented with transducers to measure the pressure and the valve spool position. Hardware for the control of each axis is described in [6]. Closed-loop control of all axes is achieved by four proprietary M2000 Programmable Servo Controllers (PSC), digital controllers that are commanded and coordinated by an industrial IBM-compatible personal computer (PC). The three M2000 axis controllers are enabled by an on/off switch. Each module controls two axes. The PC communicates with the digital controllers through a Control Area Network (CAN) bus, and issues track velocity commands, and position set-points to the other axes. Protection and safety issues are handled by the axis controllers. At this time, system motion commands are input via a joystick that is interfaced to the PC.

The arm position, valve voltage, and load pressure responses in free space with the use of the proposed sliding mode controller are depicted in Figure 5. Very accurate tracking can be observed. To demonstrate robust tracking in constrained motions, Figure 6 shows the arm position and valve control voltage responses when digging "sandy loam" according to a prescribed target impedance [2] of the bucket tip. Figure 7 shows the boom tracking response of a prescribed pattern during a typical excavation task: scooping soil and loading it to a truck. Experiments have verified the validity and feasibility of our proposed robust control schemes for autonomous operations of the robotic excavator.
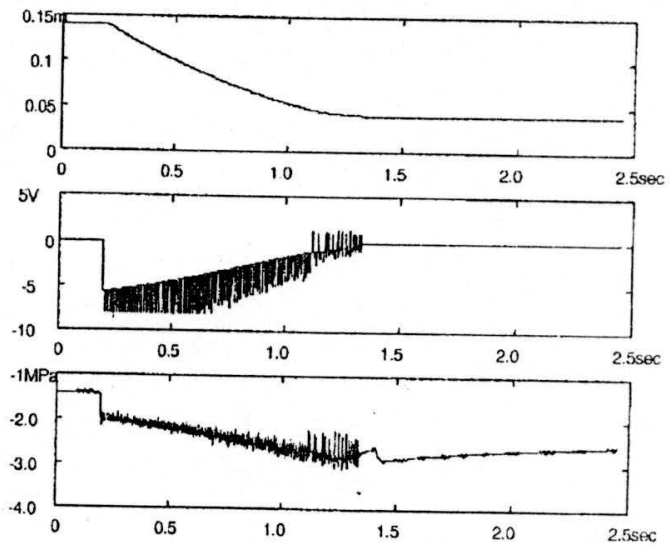

Figure 5. Experimental free motion responses.
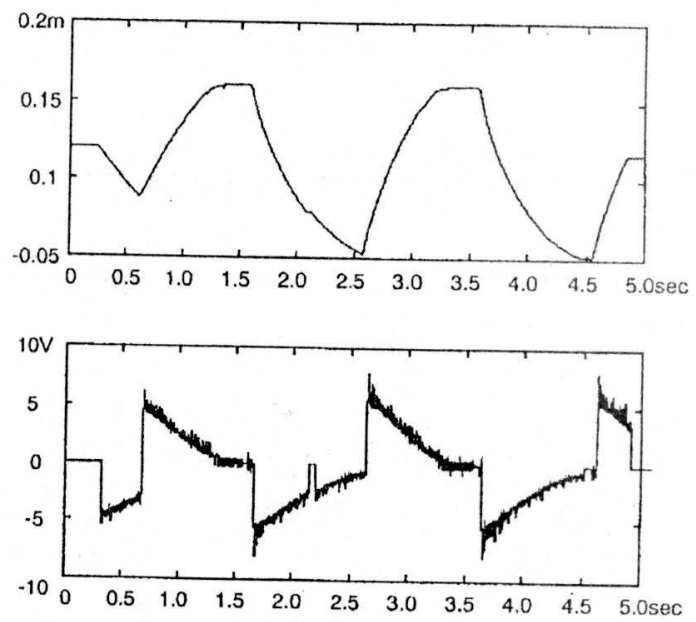

Figure 6. Experimental tracking responses when digging "sandy loam".

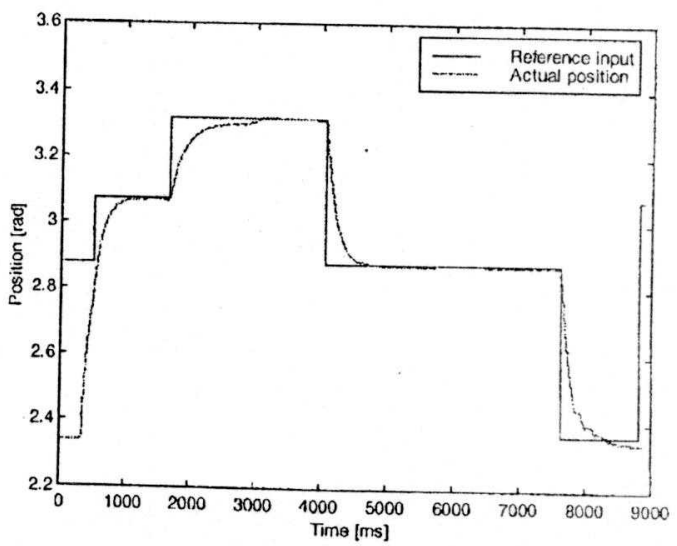

Figure 7. Experimental boom tracking response. 


\section{CONCLUSION}

In impedance control of hydraulic excavators the piston position and ram force of each hydraulic cylinder for the axis control of the boom, arm, and bucket can be determined. The problem is thus how to find the control voltage applied to the servovalves to track these desired commands to the hydraulic systems. This paper has presented the methodology, design and results of robust controllers for force and position control of electrohydraulic servo systems of a robotic excavator. Sliding mode control incorporating a fuzzy tuning approach has been successfully implemented. High performance and strong robustness are achieved in simulation of electrohydraulic servo systems for the control of working attachment axes of a robotic excavator. Experiments and field tests have validated the results obtained. Future work will concentrate on the three principal themes of the on-going project: behavioural control, environment sensing and planning.

\section{Acknowledgments}

Support of the Australian Research Council, of NS Komatsu Pty. Ltd., and of the Centre for Mining Technology and Equipment (CMTE) is gratefully acknowledged.

\section{REFERENCES}

[1] A.T. Le, Q. Nguyen, Q.P. Ha, D.C. Rye, H.F Durrant-Whyte, M. Stevens, and V. Boget, "Toward autonomous excavation", Proceedings of the International Conference on Field and Service Robotics (FSR 97), pp. 121-126, Canberra, Australia 1997.

[2] Q.P. Ha, H.Q. Nguyen, D.C. Rye, and H.F. Durrant-Whyte, "Robust impedance control of excavator dynamics", Proceedings of the International Conference on Field and Service Robotics (FSR 99), Pittsburg, USA, 1999. (in press).

[3] H.E. Merritt, Hydraulic control systems. Wiley, New York, 1976.

[4] S. Tazafoli, S., Lawrence P.D., S.E. Salcudean, D. Chan, and C.W. de Silva, "Parameter estimation and actuator friction analysis for a mini excavator", Proceedings of the IEEE International Conference on Robotics and Automation, pp. 329-334, 1996.

[5] S. Tazafoli, C.W. de Silva, and P.D. Lawrence, "Tracking control of an electrohydraulic manipulator in the presence of friction", IEEE
Trans. on Control Systems T'echnology, vol. 6 , No. 3, 1998, pp. 401-411.

[6] Q.P. Ha, Q.H. Nguyen, D.C. Rye, and H.F. Durrant-Whyte, "Sliding mode control with fuzzy tuning for an electrohydraulic position servo system", Proceedings of the IEEE International Conference on Knowledge-based Intelligent Electronic Systems (KES 98), Adelaide, Australia, Vol. 1, pp. 141-148, 1998.

[7] Q.H. Nguyen, Q.P. Ha, D.C. Rye, and H.F. Durrant-Whyte, "Feedback linearisation control for electrohydraulic systems of a robotic excavator", Proceedings of the Australian Conference on Robotics and Automation (ACRA 99), Brisbane, Australia, pp. 190-195, 1999.

[8] B. Friedland and Y.J. Park, "On adaptive friction compensation", IEEE Trans. on Automatic Control, Vol. 37, No. 10, 1992, pp. 1609-1612.

[9] C. Edwards and S.K. Spurgeon, "On the development of discontinuous observers", Int. $J$. Control, Vol. 59, No. 5, 1994, pp. 1211-1229.

[10] V.I.Utkin, Sliding modes in C'ontrol optimisation. Springer-Verlag, 1992, pp. 206222.

[11] Q.P. Ha, D.C. Rye, and H.F. Durrant-Whyte, "Fuzzy moving sliding mode control with application to robotic manipulators". Automatica, Vol. 35, N0. 4, pp. 607-616, 1999.

[12] C.L. Hwang and C.H. Lan, "The pusition control of electro-hydraulic servomechanism via a novel variable structure control". Mechatronics, Vol. 4, No. 4, 1994, pp. 369-391.

[13] I. Tunay and O. Kaynak, "Provident control of an electro-hydraulic servo with experimental results". Mechatronics, Vol. 6, No. 3, 1996, pp. 249-260.

[14] C.L. Hwang, "Sliding mode control using timevarying switching gain and boundary layer for electro-hydraulic position and differential pressure control". IEE Proc.-Control Theory Appl., Vol. 143, no. 4, 1996, pp. 325-332.

[15] Q.P. Ha, "Sliding performance enhancement with fuzzy tuning," IEE Electronics Letters, Vol. 33, No. 16, pp. 1421-1423, 1997.

[16] A.N. Zelenin, V.J. Balovnev, and I.P. Kerov, Machines for moving the earth, A.A. Balkema/Rotterdam, 1987. (Translation from Russian). 